\title{
Évaluation de la toxicité chimique des radionucléides à vie longue sur la base des directives de qualité pour l'eau de boisson fixées par l'OMS
}

\author{
V. RENAUD-SALIS ${ }^{1}$, F. MENETRIER ${ }^{2}$, A. LEUDET $^{3}$, A. FLURY-HERARD ${ }^{4}$
}

(Manuscrit reçu le 17 janvier 2002, accepté le 13 septembre 2002)

RÉSUMÉ L'estimation qui est faite actuellement des risques sanitaires liés à la gestion des déchets nucléaires à vie longue est incomplète si elle ne tient compte que des aspects radiotoxiques. Bien que cette approche soit justifiée à l'égard d'un grand nombre de radionucléides inventoriés, elle ne peut cependant être exclusive et généralisée : la toxicité chimique doit être considérée notamment lorsque la période radioactive d'un radionucléide excède $10^{5}$ ans. Pour évaluer la toxicité chimique ou radiologique d'un radionucléide, on peut confronter une dose de référence appliquée à la consommation d'eau de boisson $(0,1 \mathrm{mSv} / \mathrm{an})$ avec les informations toxicologiques existantes. L'organisation mondiale de la Santé l'a fait pour l'uranium naturel et recommande une valeur guide pour l'eau de boisson, fondée sur sa toxicité chimique $(2 \mu \mathrm{g} / \mathrm{l})$. La démarche adoptée par l'OMS est reprise ici en vue de montrer que la toxicité chimique potentielle d'un élément est une donnée à intégrer dans l'estimation du risque sanitaire des radionucléides à vie longue.

ABSTRACT Assessment of the chemical toxicity of long-lived radionuclides on the basis of WHO guidelines for drinking-water quality.

The current assessment of health risks related to long lived radionuclides waste management is not complete if accounting only for radiological toxicity aspects. Although such an approach is justified for a large number of radionuclides of concern, it nevertheless cannot be exclusive and generalised: the chemical toxicity should be considered for radionuclides with a radioactive half-life exceeding $10^{5}$ years. When assessing the chemical or radiological toxicity of a radionuclide, a reference dose applicated to drinking water consumption $(0.1 \mathrm{mSv} / \mathrm{year})$ can be compared to existing toxicological data. Such an approach has been used by the World Health Organization for natural uranium, for which a guideline value in drinking water derived from its chemical toxicity $(2 \mu \mathrm{g} / \mathrm{l})$ is recommended. WHO's approach is used here for illustrating that the potential chemical toxicity of an element is to be considered for assessing health risks related to long-lived radionuclides.

\footnotetext{
1 CEA, DSV/DRR/LRT, adresse actuelle : Institut de radioprotection et de sûreté nucléaire, DES/DIR, B.P. 17,92262 Fontenay-aux-Roses, France.

2 CEA, DSV/CARMIN, B.P. 17, 92262 Fontenay-aux-Roses, France.

3 CEA, DEN/DSNI, 91191 Gif-sur-Yvette, France.

${ }^{4}$ CEA, DSV-DIR, rue de la Fédération, 75015 Paris, France.
} 


\section{Introduction}

La loi 91-1381 du 30 décembre 1991 stipule que des recherches soient effectuées sur la gestion des déchets radioactifs à haute activité et à vie longue selon trois voies différentes : transmutation, stockage en profondeur et entreposage en surface. La loi précise dans son article 1 que la gestion de ces déchets doit être assurée dans le respect de la protection de la nature, de l'environnement et de la santé, en prenant en considération les droits des générations futures. La toxicité chronique ${ }^{5}$ des radionucléides à vie longue (RNVL) peut donc être considérée dans ce cadre.

Le premier danger identifié pour les RNVL est une toxicité radiologique. À côté de cette toxicité potentielle, certains radionucléides sont susceptibles d'induire une toxicité chimique. L'uranium naturel est un cas avéré (WHO, 1998). De manière générale, la toxicité se manifeste au travers d'effets dits «à seuil » ou « sans seuil» qui recouvrent respectivement les effets non cancérogènes et cancérogènes des substances chimiques ou radioactives. La question se pose de savoir sur quel type de toxicité se fonder pour limiter au mieux l'exposition des populations aux RNVL.

Les directives de 1'Organisme mondial de la santé (OMS) ${ }^{6}$ (WHO, 1994) relatives à la qualité de l'eau de boisson, portent simultanément sur la toxicité chimique et la toxicité radiologique des radionucléides prépondérants. Au-delà de l'évaluation du risque au travers de la consommation d'eau, l'OMS établit des valeurs guides sur lesquelles, entre autres, l'Union européenne s'appuie pour élaborer des critères de qualité des eaux de consommation humaine (Directive 98/83/CE, 1998 ; AFSSA, 2001).

Parmi les milliers de polluants impliqués dans la qualité de l'eau de boisson, une importance relative est accordée aux agents chimiques qui engendrent des effets secondaires à une exposition prolongée. Compte tenu du nombre potentiel de substances concernées, l'OMS établit des valeurs guides pour « les substances qui sont considérées comme potentiellement dangereuses pour la santé, celles que l'on rencontre relativement fréquemment dans l'eau de boisson et celles qui sont présentes à des concentrations relativement élevées »(WHO, 1994). Les plus préoccupantes sont à l'heure actuelle, les substances cancérogènes et les polluants ayant des propriétés toxiques cumulatives comme les métaux lourds (Tab. I). L'OMS distingue les substances radioactives, des polluants chimiques; l'évaluation et la gestion du risque de cancer étant différentes pour ces deux types de polluants (Brown, 1992 ; Santé Canada, 1998).

\footnotetext{
5 La toxicité chronique correspond aux effets d'une administration réitérée à long terme et à faible dose.

6 La qualité de l'eau définie par les Directives de qualité pour l'eau de boisson la rend propre à la consommation humaine et à tous les usages domestiques, dont l'hygiène personnelle.
} 


\section{TABLEAU I}

Substances chimiques inorganiques dont la présence dans l'eau revêt une importance sanitaire (WHO, 1996, 1998).

Inorganic constituents of health significance in drinking water (WHO, 1996, 1998).

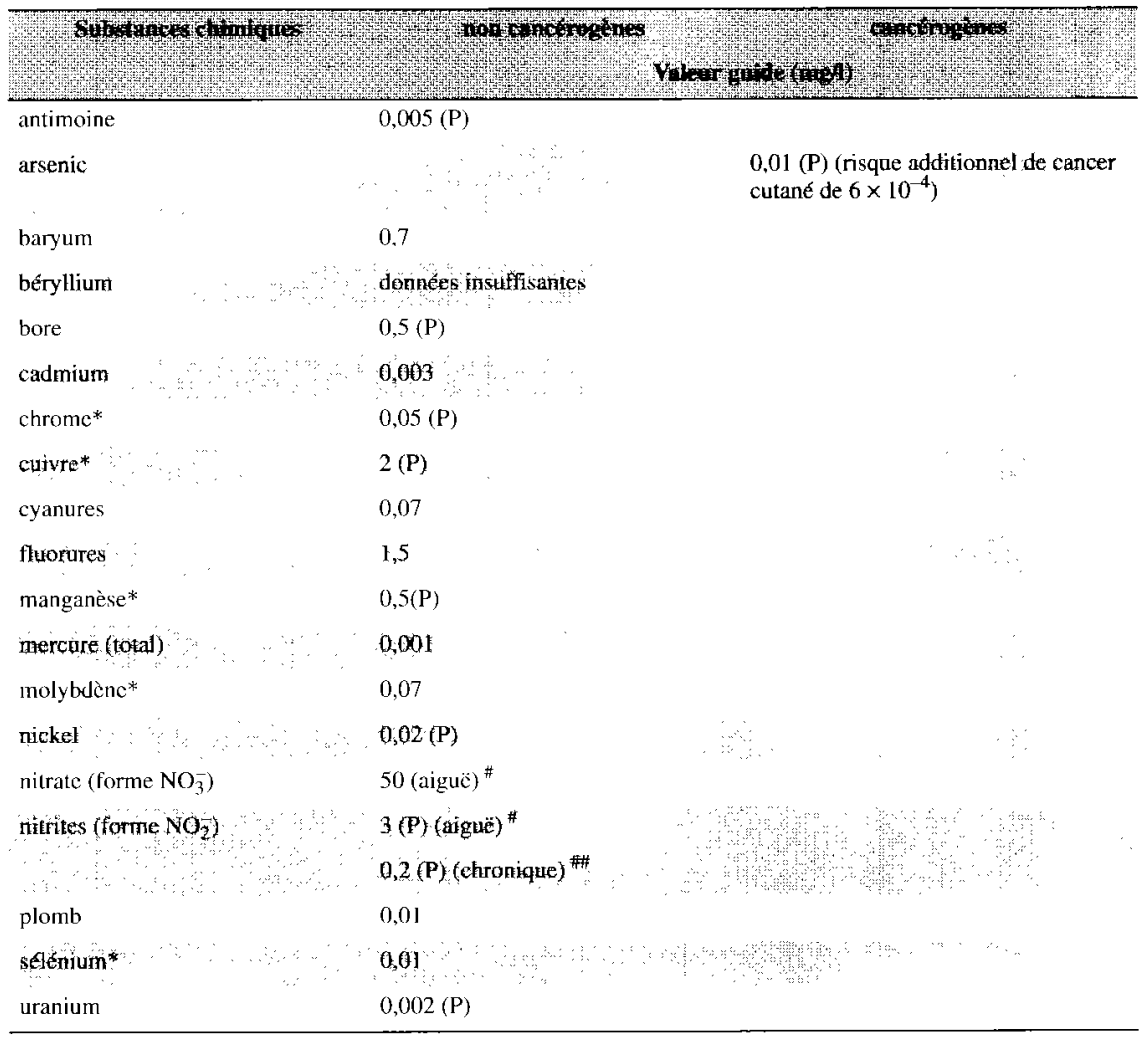

*Éléments essentiels, (P) provisoire, " valeur protectrice contre l'effet aigu de méthémoglobinémie, \#\# valeur protectrice des effets obscrvés à long terme chez l'animal (changements morphologiques au niveau du coeur, du poumon et des surrénales).

Chaque valeur guide correspond à la concentration d'un composant qui ne représente aucun risque pour la santé de l'homme qui consommerait l'eau en question pendant toute sa vie. Toutefois, un dépassement momentané de cette valeur ne signifie pas que l'eau devienne impropre à la consommation, la durée et l'amplitude des écarts sans incidence sur la santé dépendant de chaque substance. À l'opposé, certaines substances sont des nutriments essentiels et un apport insuffisant produirait un syndrome de carence. Les valeurs guides ne doivent pas être interprétées comme des limites de danger. Elles constituent des niveaux de 
référence à partir desquels les moyens nécessaires qui garantissent qu'elles sont contrôlées doivent être mis en cuvre.

\section{Les aspects chimiques de la qualité de l'eau de boisson : « effets cancérogènes », « effets non cancérogènes »}

Les méthodes qui concourent à la caractérisation du risque des «effets cancérogènes » et « non cancérogènes » sont différentes (Johannsen, 1990 ; IPCS, 1994) et seront, de ce fait, développées séparément. En revanche, elles ont en commun le fait de reposer sur les hypothèses les plus conservatrices.

- Pour les effets cancérogènes ${ }^{7}$, on admet que les effets peuvent survenir quel que soit le niveau d'exposition. On suppose donc que la plupart des cancérogènes n'ont pas de dose seuil, en deçà duquel l'effet serait nul. De ce fait, l'évaluation du risque repose sur des modèles probabilistes et s'exprime de manière quantitative par une relation entre la dose et le risque, probabilité d'apparition de cancer.

- Pour les effets non cancérogènes, on admet l'existence d'un seuil en deçà duquel aucun effet délétère ne survient. La méthode d'estimation du risque ne repose que sur des critères qualitatifs car seule la valeur du seuil est retenue ; la relation entre la dose et la réponse n'est prise en compte que pour déterminer le niveau du seuil, ce dernier étant spécifique d'un effet recherché pour une forme chimique donnée d'un élément.

Les données disponibles qui sont à la base du calcul des valeurs guides sont essentiellement l'expérimentation animale, parfois les études épidémiologiques. Les études sur les populations sont à privilégier, mais leur interprétation est souvent limitée en raison d'incertitudes sur les expositions et sur les effets, pour les faibles niveaux. Les études sur les animaux posent le problème de la représentativité de l'espèce et les limites tiennent généralement à la spéciation (généralisation des résultats à partir de l'administration d'une ou de quelques formes physico-chimiques de la substance) et aux fortes doses administrées en contraste avec les faibles niveaux auxquels les populations sont exposées. Il faut être conscient qu'à tous les niveaux d'établissement d'une valeur guide intervient le jugement d'expert dans le traitement qualitatif et quantitatif de l'estimation du risque. Les valeurs guides ne sont donc pas immuables et évoluent en fonction des progrès techniques et scientifiques. Par exemple, lorsque les données sont jugées

\footnotetext{
7 Dans le cas où une substance cancérogène n'aurait pas d'effet génotoxique avêté, l'OMS considère qu'i] existe un seuil et recommande une approche par la dose journalière tolerable pour calculer la valeur guide.
} 
insuffisantes pour établir une valeur guide, celle-ci est qualifiée de provisoire ${ }^{8}$ et fait l'objet de révisions périodiques.

\subsection{Les effets « non cancérogènes »}

L'exposition à un polluant chimique « non cancérogène » peut induire une variété d'effets toxiques dont on observe qu'ils ne s'expriment qu'au-delà d'une concentration seuil (sur un organe particulier, un système ou une fonction biologique). Ces effets varient selon la dose, la durée et la fréquence de l'exposition, mais également avec la forme physico-chimique, l'espèce animale, l'état physiologique ainsi que l'âge et le sexe de la population exposée. Ils peuvent se manifester à des intensités et des niveaux différents de l'organisme entier : moléculaires, cellulaires, et tissulaires et de leurs interactions. L'identification de ces effets est obtenue par la collecte et l'obtention de données toxicologiques les plus pertinentes (rapports publiés dans la littérature scientifique ouverte, renseignements fournis par les gouvernements, les industriels...). À l'issue d'une évaluation toxicologique approfondie, la plus basse des doses sans effet indésirable observé (DSEIO) est conservée, si possible à partir d' «étude clé » de longue durée établie par voie d'ingestion. Une dose « seuil " applicable à l'homme «dose journalière tolérable » (DIT) peut être établie, à partir de la DSEIO, le cas échéant de la dose minimale ayant un effet observé $(D M E I O)^{9}$, divisée par une série de facteurs d'incertitude $(F I)$ associés à ce seuil :

$$
D J T(\mathrm{mg} / \mathrm{kg} / \mathrm{jour})=\frac{(D S E I O \text { ou } D M E I O)}{F I}
$$

avec :

$D S E I O=$ dose sans effet indésirable observé. Dose la plus élevée d'une substance pour laquelle il n'existe pas d'augmentation significative de l'effet critique (indicateur de toxicité le plus sensible) dans la population exposée par rapport à la population témoin (sur l'espèce animale la plus sensible) ;

DMEIO = dose minimale avec effet indésirable observé. Dose la plus faible d'une substance pour laquelle il existe une augmentation significative d'un effet indésirable donné entre la population exposée et la population témoin (modèle expérimentale, études épidémiologiques);

\footnotetext{
8 Ce terme est utilisé dans les cas suivants :

a. il y a des raisons de penser qu'il existe un risque potentiel, mais les informations sont limitées dans ce sens,

b. le facteur d'incertitude est supérieur à 1000 ,

c. les substances pour lesquelles la valeur guide calculée serait inférieure à la límite de détection ou au niveau pouvant ètre atteint grâce aux méthodes de traitement normalement utilisables.

${ }^{9}$ Lorsqu'on utilise une $D M E I O$ au lieu d'unc $D S E I O$, on applique généralement un facteur d'incertitude supplémentaire.
} 
$F I=$ facteurs $d$ 'incertitude. Chaque facteur, d'une valeur de 1 à 10 , est associé à une source d'incertitude différente.

Les facteurs d'incertitude portent essentiellement sur la variabilité inter-espèces, les différences de sensibilité à l'intérieur de la population humaine, la fiabilité des données, la nature et la gravité de l'effet (effets fonctionnels, nature de la maladie en termes de viabilité, réversibilité etc.). En pratique, un facteur 100 est fréquemment appliqué, la majorité des études étant conduite sur animal de laboratoire. Cependant, les comités d'experts de l'OMS ont été amenés à constituer des facteurs de 10 à 2000 . Lorsque les données sont disponibles chez l'homme, elles peuvent cautionner un facteur plus faible. Si les données indiquent un effet tératogène potentiel, un facteur d'incertitude supplémentaire pourra être affecté, etc. Les facteurs d'incertitude sont appliqués au cas par cas. Ils peuvent varier d'un organisme de réglementation à un autre mais ce qui importe, c'est que leurs critères de choix soient toujours clairement exposés.

La valeur guide $(V G)$ applicable à la qualité des eaux de consommation est dérivée de la $D J T$ :

$$
{ }^{10} V G(\mathrm{mg} / \mathrm{l})=\frac{D J T \times P_{C} \times P}{C}
$$

avec :

$P c=$ poids corporel, paramètre standard OMS fixé à $60 \mathrm{~kg}$ pour un adulte,

$P=$ proportion de la $D J T$ attribuée à l'eau de boisson,

$C=$ consommation journalière d'eau de boisson. Elle est fixée à $21 /$ jour pour un adulte qui vit en zone tempérée. Pour ce qui concerne l'enfant $(10 \mathrm{~kg})$ et le nourrisson $(5 \mathrm{~kg})$, leur consommation respective est estimée à $11 /$ jour et $0,75 \mathrm{l} /$ jour.

Cette valeur prend en compte toutes les sources d'exposition en ne conservant que la part relative à l'eau de boisson, après détermination des concentrations moyennes de la substance dans les différents milieux : aliments, eau de boisson, air et sol (IPCS, 1994). En l'absence de ces données, le pourcentage de l'apport total ingéré avec l'eau de boisson est fixé arbitrairement à $10 \%$ (WHO, 1994). Il convient de souligner que ce pourcentage peut-être réévalué en fonction des données spécifiques dont les différents pays disposent ( $c f$. paragraphe 4.1, l'uranium).

\footnotetext{
${ }^{10}$ Les paramètres standards (consommation d'eau de boisson et poids corporel) qui sont introduits dans le calcul des valcurs guides s'ecartent des standards que la CIPR 23 (ICRP, 1975) recommande d'utiliser. Ils peuvent donc être réajustés en fonction des estimations nationales.
} 
Les valeurs guides laissent donc généralement une marge de sécurité importante (valeurs seuils, facteurs d'incertitudes) par rapport aux niveaux d'observation des effets indésirables.

\subsection{Les effets « cancérogènes »}

L'OMS reconnaît le pouvoir cancérogène des substances à partir de la classification qualitative du Centre international de recherche sur le cancer (CIRC, 1995). Les substances susceptibles d'induire un cancer par un mécanisme génotoxique sont distinguées. Dans ce cas, le mécanisme, défini comme l'interaction d'une substance, ou de ses métabolites actifs, avec le matériel génétique des cellules somatiques, est supposé sans seuil (sans que le fait soit établi). On adopte donc l'hypothèse prudente qu'un risque d'apparition de cancer existe aux plus faibles doses, sans qu'aucun effet ne soit observé ou observable, en extrapolant la relation dose-effet observée à des doses élevées, au moyen du modèle mathématique le plus pertinent (European commission, 1996; NCRP, 1989). Quel que soit le modèle utilisé, il est souhaitable qu'il fournisse une indication de toute incertitude, les risques réels d'une exposition à de faibles concentrations pouvant être 10 à 100 fois plus faibles que ceux extrapolés.

La présentation la plus fréquente qui est faite de l'estimation du risque de cancer est l'excès de risque individuel pour la vie entière ou l'excès du nombre annuel de cas de cancers attendus. L'OMS présente les valeurs guides comme étant « la concentration dans l'eau de boisson associée à un risque additionnel de cancer de $10^{-5}$ pour la vie entière (un cas supplémentaire pour 100000 personnes dans une population qui consommerait pendant 70 ans une eau de boisson contenant la substance en question à une concentration égale à la valeur guide) ». La valeur guide correspondant à un facteur de risque de $10^{-5}$ n'est cependant pas toujours applicable, notamment lorsque des raisons analytiques ou de traitements des eaux, s'y opposent. Dans le cas où la valeur guide serait établie à un niveau matériellement plus réaliste, on admet qu'elle puisse être réduite ou augmentée pour respecter des facteurs de risque compris entre $1 \times 10^{-4}$ et $1 \times 10^{-6}$, ce que certaines études ou réglementations ont adopté. Lorsqu'il n'est plus possible de respecter cet intervalle, une valeur guide provisoire est proposée et le risque de cancer associé est alors calculé. C'est le cas pour l'arsenic ${ }^{11}$ (WHO, 1994, 1998). À partir d'une enquête épidémiologique effectuée à Taiwan, choisie comme étude clé, l'EPA a estimé qu'un risque additionnel de cancer de la peau de $10^{-5}$ sur la vie entière pouvait être associé à l'ingestion d'une concentration d'arsenic de $0,20 \mu \mathrm{g} / \mathrm{l}$

"Les composés inorganiques de l'arsenic sont classés dans le groupe I du CIRC « agent cancérogène pour l'homme ». 
dans l'eau de boisson ${ }^{12}$, selon l'application d'un modèle à étapes linéaire quadratique. L'OMS a jugé d'une part que la valeur de concentration était irréaliste en comparaison des teneurs en arsenic des eaux naturelles (entre 1 et $2 \mu \mathrm{g} / \mathrm{l}$ ), d'autre part que le risque réel de cancer de la peau était surestimé, ce qui l'a conduit à proposer une valeur guide provisoire de $10 \mu \mathrm{g} / \mathrm{l}(c f$. Tab. I) correspondant à un risque additionnel de $6 \times 10^{-4}$. Le bien fondé de cette valeur a été discuté au sein de l'Académie de médecine (Boudène, 2001).

\subsection{Cas des mélanges}

L'OMS considère que les valeurs guides comportent a priori un facteur d'incertitude suffisamment important, permettant de s'abstraire d'interactions potentielles. Sauf indication contraire, on considère généralement que les effets sont additifs lorsque les composants du mélange ont des effets toxiques analogues en raison de modes d'action semblables. La question de l'évaluation de la toxicité du mélange se pose lorsque les composants atteignent des concentrations proches de leurs valeurs guide respectives.

\section{Les aspects radiologiques de la qualité de l'eau de boisson : « effets aléatoires »}

Les effets radiologiques sont traités selon les recommandations de la publication 60 de la CIPR (ICRP, 1990). L'effet biologique potentiel le plus important est l'induction de cancer dans le cas d'une exposition prolongée aux faibles doses. Il s'agit donc, selon les hypothèses de base, d'un effet sans seuil pour lequel on admet une relation dose-effet linéaire. Ce modèle est issu des données humaines (la principale source provenant d'études sur les survivants des explosions atomiques d'Hiroshima et de Nagasaki) et le risque de cancer mortel sur la vie entière est évalué à $5 \times 10^{-2} \mathrm{~Sv}^{-1}$ pour la population générale. La limite de dose annuelle établie pour le public est de $1 \mathrm{mSv}$. En radioprotection, la limite de dose est applicable à la somme des expositions résultant de toutes les pratiques réglementées, sans tenir compte des expositions naturelles, quel qu'en soit le niveau. Alors que pour chaque élément chimique, on ne peut pas dissocier ce qui revient au naturel et ce qui a été surajouté par les activités humaines, la limite individualisée correspondant à une valeur pour laquelle aucune augmentation significative de l'effet indésirable le plus sensible n'est observée (NCRP, 1989 ; ICRP, 1990 ; Santé Canada, 1998). La limite de dose «CIPR » représente la valeur maximale au-dessous de laquelle il est recommandé de maintenir l'exposition ajoutée au fond naturel, en la réduisant dans toute la mesure du possible.

\footnotetext{
${ }^{12}$ Cette donnée présentée sous la forme " excès de risque unitaire " ou « slope facteur » est disponible à partir de la base de données toxicologiques « Integrated Risk information System » (IRIS). Le registre IRIS contient des informations sur plus de 500 substances chimiques qu'il convient de comparer à d'autres bases existantes.
} 
Pour ce qui concerne la contamination par ingestion d'eau de boisson, la CIPR a fixé dans le passé, des concentrations maximales admissibles par radionucléide associées aux limites de dose pour les travailleurs (ICRP, 1959). Cette démarche a été abandonnée, faute de données suffisantes pour estimer de façon fiable cette voie d'exposition. Actuellement, l'OMS recommande une dose totale indicative de $0,1 \mathrm{mSv}$ par an ${ }^{13}$, résultant de l'ingestion de tous les radionucléides présents dans l'eau de boisson (naturels et anthropiques), qu'elle justifie comme «valeur représentant moins de $5 \%$ de la dose efficace annuelle moyenne attribuable au rayonnement de fond naturel $\gg$ compte tenu de la contribution de l'eau de boisson à l'exposition totale qui est très faible.

Dans sa publication de 1994, l'OMS recommande le calcul d'une concentration de référence $(\mathrm{g} / \mathrm{l})$, à partir de la dose totale indicative de $0,1 \mathrm{mSv}$, en fonction des doses par unité d'incorporation $(D P U I)^{14}$, des activités massiques des isotopes et d'un apport quotidien en eau évalué à 2 litres :

$$
\text { Concentration de référence }(\mathrm{g} / \mathrm{l}): \frac{1 \times 10^{-4}(\mathrm{~Sv} / \mathrm{an})}{730(\mathrm{l} / \mathrm{an}) \times\left[\sum C_{i} \times D P U I_{i} \times A_{i}\right]}
$$

avec :

$C_{i}=$ teneur isotopique de l'élément (rapport de la masse de l'isotope $i$ sur la masse de l'élément),

$D P U I_{i}=D P U I$ adulte par ingestion de l'isotope $i(\mathrm{~Sv} / \mathrm{Bq})$,

$A_{i}=$ activité massique de l'isotope $i(\mathrm{~Bq} / \mathrm{g})$.

Dans le cas de l'uranium naturel $\left[{ }^{238} \mathrm{U}(99,28 \%), \quad{ }^{235} \mathrm{U}(0,718 \%)\right.$, $\left.{ }^{234} \mathrm{U}(0,0056 \%)\right]$, on obtient une teneur limite équivalente de l'ordre de $140 \mu \mathrm{g} / \mathrm{l}$ (l'utilisation des DPUI de la publication 72 de la CIPR (ICRP, 1996) conduit à une valeur de $110 \mu \mathrm{g} / \mathrm{l})$.

\section{4. Évaluation de la toxicité chimique des radionucléides à vie longue}

\subsection{L'uranium}

La néphrotoxicité chimique induite par l'uranium est connue depuis des décennies (ICRP, 1959). Cependant l'OMS recommandait, en 1994, de retenir une valeur

\footnotetext{
${ }^{13}$ Les seuils de dépistage recommandés par l'omS sont de $0,1 \mathrm{~Bq} / 1$ pour la radioactivité $\alpha$ globale et de $1 \mathrm{~Bq} / 1$ pour la radioactivité $\beta$ globale (ne concernent pas le ${ }^{222} \mathrm{Rn}$ et ses descendants, le ${ }^{40} \mathrm{~K}$ et le tritium).

${ }^{14}$ L'OMS utilise les valeurs de DPUI établies par le National Radiological Protection Board (NRPB) de Grande-Bretagne, applicables aux adultes. Les valeurs de DPUI de la publication 72 de la CIPR (ICRP, 1996) n'ont été disponibles qu'à partir de 1996, soit deux ans après la publication de l'OMS.
} 
limite de $140 \mu \mathrm{g} / \mathrm{l}$, fondée sur des critères radiologiques, en attente d'une évaluation plus approfondie de la toxicité chimique de l'uranium. Cette perspective a été partiellement atteinte avec les résultats d'une étude subchronique (91 jours), réalisée chez le sexe et l'espèce animale les plus sensibles, permettant d'établir une $D M E I O$ pour des lésions cellulaires dégénératives du rein (Gilman et al., 1998). La valeur guide fondée sur cette « étude clé » est de $2 \mu \mathrm{g} / \mathrm{l}$ (avec un FI de 100 et un pourcentage arbitraire de $10 \%$ de l'apport total en eau de boisson) (WHO, 1998). Toutefois, la valeur actuelle est provisoire dans la mesure où aucune étude chronique adéquate n'a pu être identifiée et la relation dose-effet n'est pas parfaitement établie. De plus, des réserves sont émises quant aux performances techniques d'élimination de l'uranium pour des eaux dont la teneur est naturellement riche, pouvant atteindre dans certaines régions plusieurs centaines de microgrammes par litre.

Selon l'approche proposée par l'OMS, on constate que la toxicité de l'uranium est d'autant plus dominée par des effets d'origine chimique que les activités massiques des isotopes sont faibles. Les résultats du tableau II attestent ces faits pour l'uranium-238 et l'uranium-235. On dispose ainsi d'un moyen d'évaluer, dans le cadre des recommandations de l'OMS, la part relative de la toxicité chimique des radionucléides.

\subsection{Les radionucléides à vie longue}

I] est intéressant de poursuivre la démarche appliquée à l'uranium, plus généralement aux radionucléides à vie longue produits dans les réacteurs nucléaires électrogènes (Rapport CEA, 2000). La conversion en masses équivalentes à $0,1 \mathrm{mSv}$ apporte un éclairage sur les niveaux de concentrations de référence pouvant être atteints, par élément, dans les eaux de consommation (sans considérer d'autres facteurs limitant: solubilité, etc.) et permet de les confronter à des données toxicologiques connues, en l'occurrence les valeurs guides. L'inventaire établi par le CEA comporte 90 radionucléides; on n'a donc retenu que ceux dont la concentration de référence est supérieure ou de l'ordre de $1 \mu \mathrm{g} / \mathrm{l}$ (valeur guide la plus basse établie pour le mercure, $c f$. Tab. I). Le tableau II montre que les valeurs guides de l'uranium-238, du plomb-205 et de l'uranium235 sont atteintes avant les concentrations de référence de chacun. Cette application par radionucléide n'est cependant pas représentative des déchets à vie longue, composés d'éléments répartis en isotopes stables et/ou radioactifs. Le calcul précédent est donc reporté par élément (UOX, $45 \mathrm{GWj} / \mathrm{t}$ à 100 ans) pour lesquels on dispose de données sur la répartition isotopique dans les éléments combustibles irradiés (matrice combustible et structure métallique, Tab. III). D'après ces résultats, le césium et le thorium dont la concentration de référence est 


\section{TABLEAU II}

Évaluation de la contribution de la toxicité chimique à l'impact sanitaire sur la base de la dose de référence de $0,1 \mathrm{mSv}$; application aux radionucléides à vie longue (rapport CEA, 2000) ${ }^{15}$.

Evaluation of chemical toxicity contribution to health impact on the basis of a reference of dose $0.1 \mathrm{mSv}$; application to long-lived radionuclides (CEA report, 2000).

\begin{tabular}{|c|c|c|c|c|}
\hline $\begin{array}{c}\text { Radionucléides à vie } \\
\text { longue (teneur } \\
\text { isotopique égale à } 1 \text { ) }\end{array}$ & $\begin{array}{l}\text { Activité massique } \\
(\mathrm{Hq} / \mathrm{g})\end{array}$ & $\begin{array}{c}\text { DPUI adulte } \\
\text { Ingestion }(\mathrm{Sv} / \mathrm{Bq})\end{array}$ & $\begin{array}{l}\text { Concentration } \\
\text { de référence } \\
(0,1 \mathrm{mSv})(1 \mathrm{~g} /)\end{array}$ & 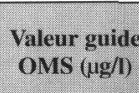 \\
\hline${ }^{123} \mathrm{Te}(\mathrm{PF})$ & $1,08 \times 10^{1}$ & $4,4 \times 10^{-9}$ & $3 \times 10^{6}$ & - \\
\hline${ }^{87} \mathrm{Rb}(\mathrm{PF})$ & $3.20 \times 10^{3}$ & $1,5 \times 10^{-9}$ & $3 \times 10^{4}$ & - \\
\hline${ }^{147} \mathrm{Sm}(\mathrm{PF})$ & $8,50 \times 10^{2}$ & $4,9 \times 10^{-8}$ & $3 \times 10^{3}$ & - \\
\hline${ }^{238} \mathrm{U}(\mathrm{NL})$ & $1,24 \times 10^{4}$ & $4,5 \times 10^{-8}$ & $2 \times 10^{2}$ & $2(\mathrm{P})$ \\
\hline${ }^{107} \mathrm{Pd}(\mathrm{PF})$ & $1,90 \times 10^{7}$ & $3,7 \times 10^{-11}$ & $2 \times 10^{2}$ & - \\
\hline${ }^{232} \mathrm{Th}(\mathrm{NL})$ & $4,06 \times 10^{3}$ & $2,3 \times 10^{-7}$ & $2 \times 10^{2}$ & - \\
\hline${ }^{205} \mathrm{~Pb}$ (PAI) & $4,25 \times 10^{6}$ & $2,8 \times 10^{-10}$ & $2 \times 10^{2}$ & 10 \\
\hline${ }^{53} \mathrm{Mn}$ (PAI) & $6,68 \times 10^{7}$ & $3,0 \times 10^{-11}$ & 70 & $500(\mathrm{P})$ \\
\hline${ }^{97} \mathrm{Tc}$ & $5,25 \times 10^{7}$ & $6,8 \times 10^{-11}$ & 40 & - \\
\hline${ }^{235} \mathrm{U}(\mathrm{NL})$ & $8,00 \times 10^{4}$ & $4.7 \times 10^{-8}$ & 40 & $2(\mathrm{P})$ \\
\hline${ }^{98} \mathrm{Tc}$ & $3,22 \times 10^{7}$ & $2,0 \times 10^{-9}$ & 2 & - \\
\hline${ }^{135} \mathrm{Cs}(\mathrm{PF})$ & $4,26 \times 10^{7}$ & $2,0 \times 10^{-9}$ & 2 & - \\
\hline${ }^{93} \mathrm{Zr}(\mathrm{PF} / \mathrm{PAI})$ & $9,30 \times 10^{7}$ & $1,1 \times 10^{-9}$ & 1 & - \\
\hline${ }^{236} \mathrm{U}(\mathrm{NL})$ & $2,40 \times 10^{6}$ & $4,7 \times 10^{-8}$ & 1 & $2(P)$ \\
\hline${ }^{59} \mathrm{Ni}(\mathrm{PAI})$ & $2,95 \times 10^{9}$ & $6,3 \times 10^{-11}$ & $7 \times 10^{-1}$ & 20 \\
\hline${ }^{79} \mathrm{Se}(\mathrm{PF})$ & $1.52 \times 10^{8}$ & $2,9 \times 10^{9}$ & $3 \times 10^{-1}$ & 10 \\
\hline
\end{tabular}

(PF) Produit de fission, (NL) noyaux lourds, (PAI) produit d'activation des impuretés

nettement inférieure à $1 \mu \mathrm{g} / \mathrm{l}$, ne présenteraient qu'une toxicité attribuable à leur caractère radioactif, à l'inverse du manganèse et du plomb, dont les concentrations de référence dépassent largement leurs valeurs guides respectives. De même, les fortes concentrations de référence observées pour le tellure, le rubidium et le palladium, sont a priori en faveur d'une toxicité prédominante de nature chimique, l'absence de valeur guide ne garantissant pas une totale innocuité. Par exemple, les données relatives à une toxicité chronique du tellure sont quasi inexistantes mais des études issues de l'exposition professionnelle (dépression, somnolence consécutives à de fortes expositions) (Alexander et al., 1988) et de l'expérimentation animale (troubles de la myélinisation) (Anthony et al., 1996) font suspecter qu'il pourrait être un composé dangereux pour la santé.

\footnotetext{
${ }^{15}$ Radioéléments sélectionnés dans « base de données des radionucléides à vie longue » sur la base d'une concentration de référence supérieure ou égale à $1 \mu \mathrm{g} / \mathrm{l}$.
} 
TABLEAU III

Évaluation de la contribution de la toxicité chimique à l'impact sanitaire sur la base de la dose de référence de $0,1 \mathrm{mSv}$; application aux éléments présents dans le combustible irradié, 100 ans après sortie du réacteur.

Evaluation of chemical toxicity contribution to health impact on the basis of a reference of dose $0.1 \mathrm{mSv}$; application to elements in spent fuel after a 100 years cooling.

\begin{tabular}{|c|c|c|c|c|c|}
\hline Radio-nucléide & $\begin{array}{l}\text { *Teneur isotopique (\%) } \\
\text { (inventaire UOX } \\
-45 \mathrm{GWj} / \mathrm{t} \text { a } 100 \text { ans) }\end{array}$ & $\begin{array}{l}\text { DPUI adulte } \\
\text { ingestion par unité } \\
\text { de masse (Sv/g) }\end{array}$ & $\begin{array}{l}\text { Élément } \\
\text { chimique }\end{array}$ & $\begin{array}{l}\text { Concentration } \\
\text { de référence } \\
(0,1 \mathrm{mSv})(\mu \mathrm{g} / 1)\end{array}$ & $\begin{array}{l}\text { Valeur guide } \\
\text { OMS (1g/I) }\end{array}$ \\
\hline${ }^{53} \mathrm{Mn}$ & $1 \times 10^{-7}$ & $2,00 \times 10^{-3}$ & {$[\mathrm{Mn}]$} & $7 \times 10^{+10}$ & $500(\mathrm{P})$ \\
\hline${ }^{123} \mathrm{Te}$ & $6.9 \times 10^{-4}$ & $4,70 \times 10^{-8}$ & [Te] & $9 \times 10^{+7}$ & - \\
\hline${ }^{125 \mathrm{~m}} \mathrm{Te}$ & $2,7 \times 10^{-13}$ & $5,79 \times 10^{+5}$ & & & \\
\hline${ }^{205} \mathrm{~Pb}$ & $6 \times 10^{-3}$ & $1,19 \times 10^{-3}$ & {$[\mathrm{~Pb}]$} & $2 \times 10^{+6}$ & 10 \\
\hline${ }^{87} \mathrm{Rb}$ & 66,4 & $4,81 \times 10^{-6}$ & {$[\mathrm{Rb}]$} & $4 \times 10^{+4}$ & - \\
\hline${ }^{107} \mathrm{Pd}$ & 15,7 & $7,04 \times 10^{-4}$ & {$[\mathrm{Pd}]$} & $1 \times 10^{+3}$ & - \\
\hline${ }^{93} \mathrm{Zr}$ & 0,39 & $1,02 \times 10^{-1}$ & {$[\mathrm{Zr}]$} & $3 \times 10^{+2}$ & - \\
\hline${ }^{93} \mathrm{Mo}$ & $2.6 \times 10^{-3}$ & $1,10 \times 10^{+2}$ & {$[\mathrm{Mo}]$} & 50 & 70 \\
\hline${ }^{238} \mathrm{U}$ & 98,7 & $5,60 \times 10^{4}$ & {$[\mathrm{U}]$} & 20 & $2(\mathrm{P})$ \\
\hline${ }^{236} \mathrm{U}$ & 0.53 & $1,13 \times 10^{-1}$ & & & \\
\hline${ }^{235} \mathrm{U}$ & 0,73 & $3,76 \times 10^{-3}$ & & & \\
\hline${ }^{234} \mathrm{U}$ & $4 \times 10^{-2}$ & $1,13 \times 10^{+1}$ & & & \\
\hline${ }^{232} \mathrm{U}$ & $1,3 \times 10^{-7}$ & $2,69 \times 10^{+5}$ & & & \\
\hline${ }^{79} \mathrm{Se}$ & 8,56 & $4,42 \times 10^{-1}$ & [Se] & 4 & 10 \\
\hline${ }^{63} \mathrm{Ni}$ & 0,044 & $3,13 \times 10^{+2}$ & {$[\mathrm{Ni}]$} & 1 & 20 \\
\hline${ }^{151} \mathrm{Sm}$ & 0,78 & $9,55 \times 10^{+1}$ & [Sm] & $2 \times 10^{-1}$ & - \\
\hline${ }^{147} \mathrm{Sm}$ & 25,05 & $4,16 \times 10^{-5}$ & & & \\
\hline${ }^{228} \mathrm{Th}$ & $3,4 \times 10^{-2}$ & $2,19 \times 10^{+6}$ & [Th] & $1.5 \times 10^{-4}$ & - \\
\hline${ }^{230} \mathrm{Th}$ & 85,68 & $1.60 \times 10^{+2}$ & & & \\
\hline${ }^{137} \mathrm{Cs}$ & 7,72 & $4,18 \times 10^{+4}$ & [Cs] & $4 \times 10^{-5}$ & - \\
\hline${ }^{135} \mathrm{Cs}$ & 22,55 & $8,53 \times 10^{-2}$ & & & \\
\hline
\end{tabular}

*Teneur massique du RNVL en gramme par tonne métal initial pour un combustible irradié type UOX dont le taux de combustion est de $45 \mathrm{GWj} / \mathrm{t}$ et refroidi 100 ans.

Entre ces extrêmes, les valeurs guides du molybdène, de l'uranium, du sélénium et du nickel sont dans les ordres de grandeur de leurs concentrations de référence. Il est préférable de s'en tenir à des ordres de grandeur compte tenu du caractère modulable des valeurs guides, ne serait ce qu'en fonction des données acquises en matière d'exposition environnementale. Par exemple, le Canada évalue actuellement une valeur guide de l'uranium naturel à $10 \mu \mathrm{g} / \mathrm{l}$, selon une 
estimation d'un apport quotidien par l'eau de boisson de $35 \%{ }^{16}$ (Santé Canada, 1999), tandis que les États-Unis l'évalue à $20 \mu \mathrm{g} / \mathrm{l}$ selon un pourcentage imputable à l'eau de boisson de $80 \%$ de l'apport total quotidien (EPA, 2000).

Concernant les aspects radiologiques, les valeurs de concentration de référence, calculées en fonction des $D P U I$, peuvent être discutées compte tenu des niveaux d'incertitudes associés. En effet, la CIPR recommande des valeurs de $D P U I$ qui relèvent, au cas par cas, de connaissances inégales sur les facteurs de transfert et les données biocinétiques, ces aspects étant spécifiés dans les différents rapports de la Commission, au travers des monographies. À ces incertitudes, de niveaux variables, se surajoutent celles issues des hypothèses générales admises pour l'élaboration des modèles dosimétriques, consensuellement conservatrices. Le National Council on Radiation Protection and Measurements (NCRP) a procédé dans son rapport $\mathrm{n}^{\circ} 15$ (1998) à une expertise semi-quantitative de la fiabilité des $D P U I$ d'une vingtaine de radionucléides. De cette analyse, il ressort que l'incertitude associée à la valeur de $D P U I$ peut être minime (cas du césium-137) comme s'étendre sur plusieurs ordres de grandeur (cas du palladium), si on ne considère en première approche que l'origine (observations sur l'homme ou expérimentations animales), la qualité et le nombre des études disponibles par radionucléide.

Ces observations, replacées dans le contexte de gestion actuel, montrent que l'on ne peut décider de la prépondérance du type de toxicité d'un radionucléide à vie longue que dans le cas où les valeurs seraient établies (valeur guide, $D P U I$ ) et distinctes. Dans le cas contraire, il importera d'avoir la meilleure connaissance possible des marges de sécurité par rapport au niveau de l'effet observé sur des modèles expérimentaux représentatifs. L'OMS considère la valeur la plus pénalisante de toxicité chimique ou radiologique en conservant les méthodes d'évaluation et de gestion dont elles sont issues. L'analyse conduite à partir de l'évaluation des risques chimiques et radiologiques montre qu'ils ne se prêtent pas à leur comparaison directe et que d'autres voies d'approche devraient être envisagées.

\section{Conclusion}

L'OMS recommande de fonder l'évaluation de la toxicité de l'uranium dans l'eau de boisson sur des critères chimiques qui sont plus sévères que ceux issus des recommandations internationales de radioprotection. Cette recommandation n'est pas reprise dans la nouvelle directive européenne dont la parution est antérieure à

\footnotetext{
${ }^{16}$ Cette donnée décrit l'upport pour la population générale qui cst généralement exposée à une eau dont la teneur en uranium est inférieure à $10 \mu \mathrm{g} / \mathrm{l}$.
} 
celle de l'OMS. Cependant elle est à prendre en considération dans l'estimation du risque sanitaire lié aux conséquences de la gestion des déchets nucléaires à vie longue et incite plus généralement à s'assurer que le seul respect des normes de radioprotection n'élude pas la question d'une action chimique potentielle. Les inventaires de déchets devraient considérer la masse de tous les éléments présents (sans critères d'exclusion fondés sur la période radioactive) pour plus de clarté dans les interprétations sur les effets sanitaires potentiels. De même, les niveaux de contaminants déterminés à partir des scénarios d'impact devraient toujours se référer à l'élément dans sa globalité (sans dissocier les isotopes), et être exprimés en concentration massique.

L'OMS a réalisé des évaluations toxicologiques sur des polluants qui ne couvrent pas la totalité des éléments que l'on retrouve dans les déchets nucléaires à vie longue. D'autres évaluations sont en cours, certaines seront révisées et les méthodes elles-mêmes évoluent. Tout cela relève du cas par cas et constitue un processus très long. On ne peut guère s'attendre, dans des délais raisonnables, à ce que des évaluations soient disponibles pour l'ensemble des familles d'éléments présents dans les déchets radioactifs à vie longue.

Remerciements. Les auteurs remercient vivement le docteur Jacques Lafuma (Commission nationale d'évaluation) pour son précieux concours à l'élaboration de ce document.

\section{RÉFÉRENCES}

AFFSSA (2001) Avis relatif au rapport du groupe de travail mis en place par la direction générale de la santé sur la qualité des eaux livrées à la consommation, Agence française de sécurité sanitaire des aliments, 11 décembre 2001 .

Alexander J., Thomassen Y., Aaseth J. (1988) Tellurium, Handbook on toxicity of inorganic compounds (H.G. Seiler, H. Sigel, Eds) pp. 669-674. M. Dekker, New York.

Anthony D.C., Montine T.J., Graham D.G. (1996) Toxic responses of the nervous system, Casarett et Doull's toxicology, the basic science of poisons (C.D. Klaassen, Ed) pp. 463-486. McGraw-Hill, New York.

Boudène Cl. (2001) Rapport sur le risque sanitaire lié à la présence d'arsenic dans l'eau minérale naturelle alimentant les établissements thermaux selon la concentration en arsenic de l'eau et l'orientation thérapeutique pratiquée, Bull. Acad. Nat. Méd. 185(8), 1587-1599.

Brown S.L. (1992) Harmonizing chemical and radiation risk management, Environ. Sci. Technol. 26(12), 2336-2338.

CIRC (1995) IARC Monographs on the evaluation of carcinogenic risks to humans: list of IARC evaluations, Centre International de Recherche sur le Cancer, Lyon.

Directive 98/83/CE (1998) Relative à la qualité des eaux destinées à la consommation humaine, JOCE L 330 du 5 décembre 1998, pp. 32-54.

EPA (2000) National Primary Drinking Water Regulations; Radionuclides; Notice of Data Availability, US Env. Prot. Ag. Fed. Reg. 65(7-8), $21586-21588$. 


\section{ÉVALUATION DE LA TOXICITÉ CHIMIQUE DES RADIONUCLÉIDES}

European commission (1996) Risk assessment for new notified substances and Commission Regulation (EC) $N^{\circ}$ 1488/94. Technical guidance document of Commission Directive 93/67/EEC, part I, Luxembourg.

Gilman A.P., Villeneuve D.C., Secours V.E., Yagminas A.P., Tracy B.L., Quinn J.M., Valli V.E., Moss M.A. (1998) Uranyl nitrate: 28-day and 91-day studies in the Sprague-dawley rat., Fundam. Appl. Toxical. 41, 117-128.

ICRP Publication 2 (1959) Report of Committee II on Permissible Dose for Internal Radiation. Pergamon Press, Oxford.

ICRP Publication 23 (1975) Reference Man: Anatomical, Physiological and metabolic Characterics. Pergamon press, Oxford.

ICRP Publication 60 (1990) Recommendations of the International Commission on Radiological Protection, Ann. ICRP 21(1-3).

ICRP Publication 72 (1996) Age-dependent Doses to Members of the Public from Intake of Radionuclides: part 5, Compilation of ingestion and inhalation dose coefficients, Ann. ICRP 26(1).

IPCS (1994) Assessing Human Health Risks of chemicals: Derivation of Guidance Values for Health-Based Exposure Limits, International Programme on Chemical Safety. Environmental Health Criteria 170 , WHO, Geneva.

IRIS United States Environmental Protection Agency, Substances File List: http://www.epa.gov/iris/

Johannsen F.R. (1990) Risk assessment of carcinogenic and non carcinogenic chemicals, CRC Crit. Rev. Toxicol. 20(5), 341-367.

Loi $\mathrm{n}^{\circ}$ 91-1381 du 30 décembre 1991, Relative aux recherches sur la gestion des déchets radioactifs, JORF, $1^{\mathrm{er}}$ janvier 1992,10 pages.

NCRP (1989) Comparative Carcinogenicity of lonizing Radiation and Chemicals, NCRP Report $n^{\circ} 96$, National Council on Radiation Protection and Measurements, Bethesda, MD.

NCRP (1998) Evaluating the reliability of biokinetic and dosimetric models and parameters used to assess individual doses for risk assessment purposes, NCRP Commentary $\mathrm{n}^{\circ} 15$, National Council on Radiation Protection and Measurements, Bethesda, MD.

Rapport CEA (2000) Base de données sur less radionucléides à vie longue (RNVL), Document collectif CEA-version 2, DCC 00-965.

Santé Canada (1998) Évaluation et gestion des risques de cancer associés aux rayonnements ionisants et aux agents chimiques, Commission de contrôle de l'énergie atomique, Ottawa, Ontario.

Santé Canada (1999) L'uranium dans l'eau potable: document sous consultation publique, Souscomité fédéral-provincial sur l'eau potable, Ottawa, Ontario.

WHO (1994) Guidelines for drinking-water quality, Vol. 1: Recommendations. World Health Organization, Geneva.

WHO (1996) Guidelines for drinking-water quality, Vol. 2: Health Criteria and other supporting information. World Health Organization, Geneva.

WHO (1998) Guidelines for drinking-water quality, addendum to Vol. I: Recommendations. World Health Organization, Geneva. 